\title{
Propuesta de un índice para evaluar la gestión del control interno
}

\section{Proposal of a coefficient to evaluate the management of the internal control}

\author{
Leudis Orlando Vega de la Cruz*, Yosvani Orlando Lao León \\ y Any Flor Nieves Julbe
}

Departamento de Ingeniería Industrial, Facultad de Ciencias Empresariales, Universidad de Holguín, Holguín, Cuba

Recibido el 10 de diciembre de 2015; aceptado el 30 de junio de 2016

Disponible en Internet el 6 de febrero de 2017

\section{Resumen}

Derivado de su misión humanitaria, de prestar una asistencia sanitaria de calidad a la población, en los hospitales y establecimientos sanitarios, se necesita disminuir la aparición de hechos que puedan atentar contra la integridad física y psicológica de los pacientes que atienden. Una de las estrategias a considerar para superar este reto, es la implementación adecuada del sistema de control interno, que garantice la sostenibilidad de los aseguradores, mediante el aumento de la eficiencia en la atención de las personas, factible únicamente dentro de un modelo de atención que cuantifique la gestión sobre la población ya identificada y atendida. El objetivo de este artículo fue proponer un índice de gestión del control interno para una entidad hospitalaria. Este fue concebido bajo las pautas de la modelación multicriterio en conjunto con la detección de debilidades a través de las redes de Petri. Para esto se determinó el orden de importancia y cumplimiento de los componentes del control interno y al unísono se identificó la no fiabilidad de los procesos críticos en la entidad objeto de aplicación práctica.

(C) 2017 Universidad Nacional Autónoma de México, Facultad de Contaduría y Administración. Este es un artículo Open Access bajo la licencia CC BY-NC-ND (http://creativecommons.org/licenses/by-nc-nd/4.0/).

Palabras clave: Control interno; Modelación multicriterio; Redes de Petri; Fiabilidad; Hospital

Códigos JEL: C18; C44; 118

\footnotetext{
* Autor para correspondencia.

Correo electrónico: leovega@uho.edu.cu (L.O. Vega de la Cruz).

La revisión por pares es responsabilidad de la Universidad Nacional Autónoma de México.
} 


\begin{abstract}
Derived from its humanitarian mission, to pay quality health care to the population, in hospitals and health facilities is needed reduce the occurrence of events that may threaten the physical and psychological integrity of the patients they serve. One of the strategies to consider to overcome this challenge is the proper implementation of the internal control system, to ensure the sustainability of insurers, by increasing efficiency in the care of people, feasible only within a care model that quantifies and management on population identified and addressed. The objective of this paper was to propose a coefficient to evaluate the internal control management for a hospital entity. This was conceived under the guidelines of the multicriteria modeling in conjunction with the detection of critical points through Petri nets. For this, the order of importance and fulfillment of the components of Internal Control and in unison the unreliability of critical processes in the entity being identified practical application was determined.

(C) 2017 Universidad Nacional Autónoma de México, Facultad de Contaduría y Administración. This is an open access article under the CC BY-NC-ND license (http://creativecommons.org/licenses/by-nc-nd/4.0/).
\end{abstract}

Keywords: Internal control; Multi-criteria modeling; Petri’s nets; Reliability; Hospital

JEL classification: $\mathrm{C} 18$; C44; 118

\title{
Introducción
}

La incertidumbre de los escenarios actuales en que se desarrolla la actividad empresarial, aparejado a la necesidad de responder de forma adecuada a los continuos cambios y las cambiantes demandas de los clientes, conllevan una significativa modificación en lo que a la gestión empresarial se refiere, resultando esencial en este sentido, el papel del control interno; como elemento del proceso de dirección que más contribuye a mejorar el desempeño del sistema.

En estudios recientes (Comas Rodríguez, 2013; Bolaño Rodríguez, 2014), se demuestran un conjunto de carencias relacionadas con la gestión del control interno en organizaciones cubanas, estas se centran fundamentalmente en:

1. Un limitado enfoque de proceso y hacia la mejora continua.

2. Aplicación aislada de las herramientas de control para la gestión empresarial.

3. Inoportunidad y poca flexibilidad en el proceso de toma de decisiones.

4. Ausencia de herramientas para un diagnóstico permanente.

5. Escasa fiabilidad de la información proporcionada.

6. Poca fiabilidad del proceso de toma de decisiones desarrollado por los directivos.

Especialmente las entidades pertenecientes al sector de la salud, a partir de declarar en el Juramento Hipocrático, pretenden beneficiar a los pacientes y lo sostienen como política y obligación. Sin embargo, fruto de la compleja relación entre los procesos que se desarrollan, la influencia de las tecnologías y las relaciones humanas, estas atenciones implican la alta probabilidad de ocurrencia de eventos adversos que puedan perjudicar al paciente (Rave, Trujillo, Castro y Gómez, 2014). Estos, generan un incremento de reclamaciones y (o) acciones judiciales, aparejado al sobregasto en costo global del cuidado médico.

Para dar solución a esta situación problemática se desarrolló un procedimiento que integra herramientas del control de gestión, como la evaluación de control interno, la gestión de riesgos y el enfoque por proceso. Mediante la determinación del Valor de Evaluación General de 
Administración (VEGA) en el control interno, contextualizado al marco regulatorio cubano, a través de sus cinco componentes declarados en la Resolución 60 del 2011 de la Contraloría General de la República de Cuba. Dada la diversidad de variables que inciden en su determinación, fue necesaria la introducción de la modelación multicriterio. Esta ha sido utilizada en contextos similares (Badri, Ghazanfari y Shahanaghi, 2014; Argueta, Gaytán Iniestra y Arroyo López, 2014), para la determinación del nivel de importancia de las variables en determinado objeto de estudio.

Partiendo de que el control interno pretende inspeccionar en paralelo las actividades bajo determinadas restricciones en su funcionamiento, como por ejemplo la utilización de los recursos disponibles; en este contexto se utilizó las redes de Petri como herramienta de análisis que permite identificar, modelar y priorizar estas restricciones (Hernández Cely, Leal y López, 2013; Zapata, Hoyos y Quintero, 2014; Araújo, Araújo, Medeiros y Barroso, 2015; Castro Rivera y Cuervo Oliveros, 2015; Varela, Rojas Ramírez, Hernández Gómez, Morales González y Jiménez Reyes, 2015). Para validar la propuesta se presenta una aplicación en una entidad hospitalaria cubana.

\section{Metodología}

El diseño del procedimiento resultó del análisis de la experiencia teórico-práctica de los autores y los resultados analizados en el sistema empresarial cubano. El procedimiento está formado por cuatro fases, representado en la figura 1, el cual se explicará a continuación:

\section{Fase I. Ambientación}

Objetivos: determinar los elementos vitales para el inicio de la gestión del control, definir los objetivos de la investigación, el estado del control interno de acuerdo a los controles en los últimos períodos según la guía de autocontrol y los procesos críticos de la organización para lograr pertinencia en el estudio.

\section{Definir los objetivos de la investigación}

Se definirán los objetivos específicos de las evaluaciones a realizar para verificar en qué medida se alcanza el estado deseado una vez culminada esta fase, lo que permitirá evitar el pase a la siguiente con un modelo erróneo.

\section{Contextos internos}

Se definirán en conjunto con el equipo auditor y el comité de prevención, el informe de resultados de la aplicación de la guía de autocontrol, emitida en la resolución 60 del 2011 por la Contraloría General de la República de Cuba, así como otros elementos estratégicos relacionados con el control.

\section{Selección de los expertos}

Para esto se definirá que el área del conocimiento en la que se enmarcan sus competencias sea la de «dirección» con dominio en los temas siguientes: especialista en calidad, auditoría y control de procesos, alta experiencia demostrada y diversidad de instituciones.

\section{Identificación de los procesos más trascendentales en la entidad}

Se obtendrán los pesos de cada proceso mediante las valoraciones de los expertos. Estos podrán expresar sus preferencias de dos formas: a través de un valor cuantitativo o a través de la comparación entre los criterios. Cuando los expertos emiten sus valoraciones respecto a los criterios, si 


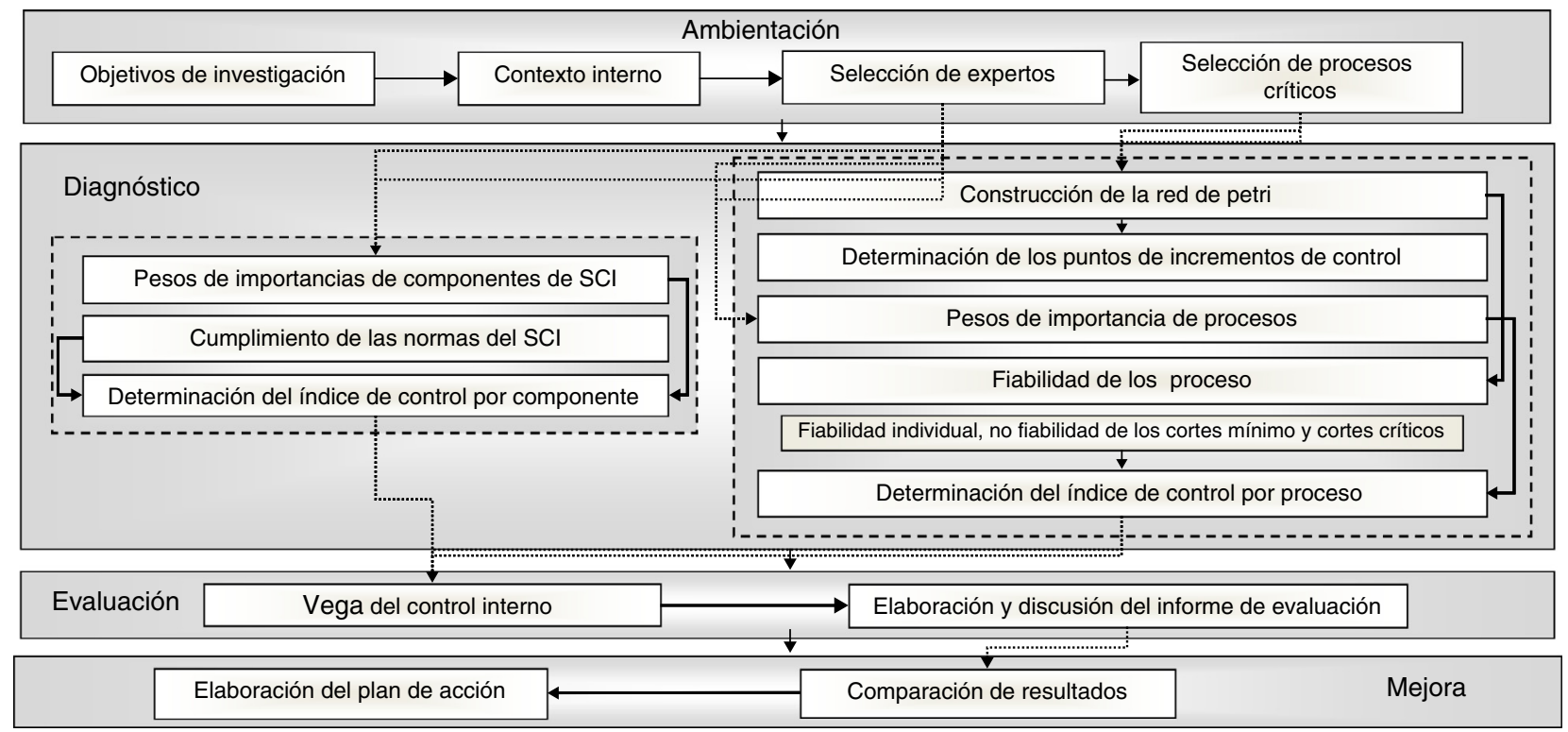

Figura 1. Procedimiento para la determinación del Valor de Evaluación General de Administración del Control Interno.

Fuente: elaboración propia. 
dos criterios tienen igual valoración indicará que ambos criterios son igualmente importantes y si un criterio tiene mayor valor que otro significará que este primero es más importante. Los valores de los pesos deberán cumplir la condición siguiente:

$$
0 \leq W_{j} \leq 1 \quad y \quad \sum_{j=1}^{j=m} W_{j}=1
$$

Donde m será la cantidad máxima de procesos y j representará el puntero de desplazamiento en la selección de un proceso dentro de la función sumatoria. Luego se aplicará el principio de Pareto, para seleccionar aquellos procesos críticos.

\section{Fase II. Diagnóstico}

Objetivo: determinar los índices de gestión del control mediante dos variantes, con el enfoque de procesos y a través de los componentes del control interno, mediante la fiabilidad e importancia de los procesos y sus componentes.

\section{Determinación de la importancia de cada componente del control interno}

Para ello se encuestará a cada experto para que emita su opinión acerca de la importancia que tiene cada componente del control interno con relación al resto de los componentes, atendiendo a diferentes criterios como: impacto en la sociedad y necesidad de mejoramiento. Se verificará que la sumatoria de estos valores relativos debe ser igual a 100 (Cornelio, Jiménez Hernández y Bron Fonseca, 2014).

\section{Determinación de las evaluaciones emitidas por los componentes}

Se harán corresponder los valores identificados en la guía para lo cual se le asignará a cada indicador su porcentaje equivalente con el promedio del componente seleccionado.

\section{Determinación del índice de control por componentes}

Se propone la aplicación del método suma ponderada, con la expresión (1) que se presenta a continuación:

$$
I C_{\text {compSCI }}=\sum_{i=1}^{m} P_{i} \times C_{i}
$$

Donde: $\mathrm{IC}_{\text {compSCI}}$ : índice de gestión del control interno por componentes; $\mathrm{P}_{\mathrm{i}}$ : peso absoluto del componente i (obtenido con anterioridad); $\mathrm{C}_{\mathbf{i}}$ : representa los valores numéricos correspondidos al cumplimiento de las normas (obtenido con anterioridad).

Con el resultado del IC se utilizará la escala definida en la tabla 1, correspondiéndose los valores de control con el estado de la organización.

\section{Construcción de la red de Petri}

A partir de (los) proceso (s) seleccionado (s) como críticos, se comenzará con la traducción de las acciones de este, a lugares y transiciones ${ }^{1}$ para la construcción de la red de Petri, como se muestra en la tabla 2.

\footnotetext{
${ }^{1}$ Referidas como tareas en la investigación.
} 
Tabla 1

Evaluación del control

Índice de control

IC $\geq 0.8$

$0.6 \leq \mathrm{IC}<0.8$

Eficiente

$0.4 \leq \mathrm{IC}<0.6$

Alto

$0.2 \leq \mathrm{IC}<0.4$

Medio

IC $<0.2$

Bajo

Deficiente

Fuente: elaboración propia.

Tabla 2

Interpretaciones para una red de Petri

\begin{tabular}{lll}
\hline Lugares de entrada & Transiciones & Lugares de salida \\
\hline Precondiciones & Eventos & Poscondiciones \\
Datos de entrada & Paso de cómputo & Datos de salida \\
Necesidad de recursos & Acciones o tarea & Recursos liberados \\
Condiciones & Cláusula lógica & Conclusiones \\
\hline
\end{tabular}

Fuente: Murillo Soto (2008, p. 110).

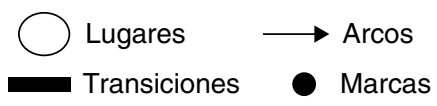

Figura 2. Elementos de una red de Petri.

Fuente: elaboración propia.

La construcción del grafo se realizará utilizando la simbología presentada en la figura 2.

\section{Determinación de puntos de control}

El propósito es evitar errores como los que se muestran ejemplificados en la figura 3 (Murillo Soto, 2010; Distéfano y Pérez, 2011; Alverca Torres y Valarezo Collahuazo, 2012; Sánchez, Herrera y Rovetto, 2014):

1. Transiciones sin condiciones de entradas y (o) salidas: impide que el proceso finalice satisfactoriamente. Las transiciones dos y cuatro no tienen lugares de entrada, ni de salida respectivamente.

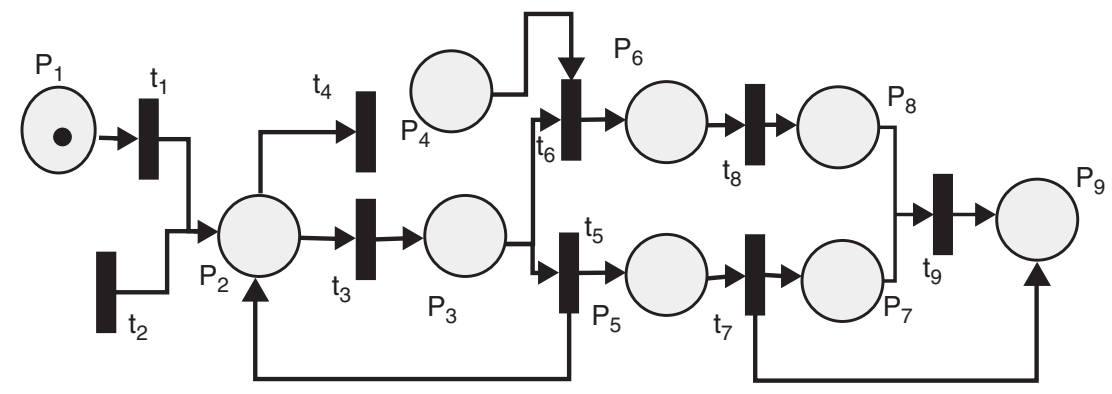

Figura 3. Incumplimientos de las propiedades estructurales.

Fuente: elaboración propia. 
2. Transiciones muertas: transiciones que nunca pueden ser finalizadas. La transición seis no cuenta con el lugar o marcado de la condición $\mathrm{P}_{4}$ por consiguiente nunca será ejecutada y estaría muerta.

3. Bloqueo: estancamiento de una transición antes de que esta alcance el final del proceso. La transición tres dispara un solo marcado al lugar tres, por lo que una de las transiciones, seis o cinco no sucederá, por lo que ocurrirá un bloqueo en el proceso y no continuará su curso.

4. Ciclos infinitos: trampa en la que una transición puede caer repetitivamente una y otra vez en un bucle sin final como es el caso de la transición cinco.

5. Actividad en ejecución después de finalizado el proceso: el objetivo final del proceso es alcanzado y luego existen transiciones que siguen ejecutándose. La transición nueve se ejecutará, cuando el marcador final sea alcanzado desde la transición siete.

6. Lugares en sitos diferentes del sitio final después de finalizado el proceso: existencia de marcados después de finalizado el proceso. El lugar nueve estará marcado cuando sea alcanzado el proceso.

\section{Determinación de la importancia de cada proceso}

De forma similar que anteriormente en este caso para los procesos y para ello se recomiendan los criterios siguientes: impacto del proceso en la sociedad, ingresos generados, madurez del sistema de información y desempeño del proceso.

\section{Determinación de la fiabilidad de los procesos}

Determinar la fiabilidad de cada tarea individual, mediante los pasos siguientes:

\section{Paso 1. Determinación de los cortes mínimos}

Un camino entre dos lugares, es cualquier sucesión de tareas y arcos, donde no se repitan tareas. Un corte $\left(\mathrm{K}_{\mathbf{i}}\right)$, se trata de un conjunto de arcos, cuyo fallo provoca el fallo del sistema (Andujar Rodríguez, García Pérez y Cruz Rembaud, 2000).

\section{Paso 2. Determinación de los cortes críticos}

Una vez identificados los caminos y cortes mínimos, se determinará la no fiabilidad como la probabilidad de que al menos un corte mínimo haya ocurrido. A partir de los caminos mínimos será posible encontrar los cortes mínimos, se determinarán, suponiendo independencia en los fallos, como la probabilidad de que fallen ambos procedimientos determinándose como el producto de las no fiabilidades de las tareas que la componen.

\section{Determinación de la fiabilidad de cada actividad}

Para la determinación de la fiabilidad del sistema, se determinará la fiabilidad de las actividades individualmente, siguiendo el método similar al propuesto en el método Program Evaluation and Review Tecnique, donde la fiabilidad estará dada por la expresión 2:

$$
F r=\frac{a+4 m+b}{6}
$$

Donde: $a$ : fiabilidad optimista, fiabilidad mínimo de ejecución de una actividad cuando todas las variables que intervienen se desarrollan excepcionalmente; $b$ : fiabilidad pesimista, fiabilidad de ejecución cuando concurren circunstancias desfavorables; $m$ : fiabilidad más probable, cuando la fiabilidad de ejecución no sufre ni circunstancias positivas ni negativas.

Para la determinación de la fiabilidad más probable, se buscará el complemento del número de veces que ocurrieron fallos en un período $\left(\mathrm{N}_{\mathbf{f}}\right)$ determinado (expresión 3), en un horizonte temporal 
dado, teniendo en cuenta que en un mismo intervalo de tiempo determinado $\left(\mathrm{N}_{\mathbf{T}}\right)$ pueden ocurrir diferentes fallos.

$$
m_{T i}=1-\frac{N f}{N t}
$$

Determinación de la no fiabilidad de los cortes

Después de calcular la fiabilidad individual de las actividades, se determinarán los cortes mínimos suponiendo independencia en los fallos, la probabilidad de que fallen ambos procedimientos es el producto de las no fiabilidades de las tareas que la componen. Los cortes críticos se determinarán seleccionando aquellas actividades cuya probabilidad de no fiabilidad sea superior al $5 \%$ (se recomienda este umbral, no obstante puede ser variado por el equipo auditor).

Paso 3. Determinación del índice de fiabilidad

El cálculo de la fiabilidad del control del proceso $\left(\mathrm{F}_{\mathrm{i}}\right)$ se determinará por la expresión 4 :

$$
F_{i}=1-\frac{N_{K}}{N_{T}}
$$

Donde: $\mathrm{N}_{\mathbf{k}}: \mathrm{n} .{ }^{\circ}$ de cortes críticos en el proceso $\mathrm{i} ; \mathrm{N}_{\mathbf{T}}$ : total de cortes mínimos en el proceso $\mathrm{i}$.

\section{Determinación del índice de control por proceso}

La determinación del índice de gestión del control por proceso, se realizará mediante la expresión 5, y su evaluación se hará según lo mostrado en la tabla 1.

$$
I C_{\text {proceso }}=\sum_{i=1}^{i=m} P_{i} \times F_{i}
$$

Donde: $\mathrm{IC}_{\text {proceso }}$ : índice de gestión del control interno por proceso; $\mathrm{P}_{\mathbf{i}}$ : peso absoluto del proceso $\mathrm{i}$ (obtenido con anterioridad); $\mathrm{F}_{\mathbf{i}}$ : representa la fiabilidad del proceso $\mathrm{i}$ (obtenido con anterioridad).

\section{Fase III. Evaluación}

Objetivo: determinar el índice de gestión del control interno mediante el VEGA del control interno.

\section{Determinación del Valor de Evaluación General de Administración del control interno}

Determinados los índices de control por componente de control interno y por procesos críticos de la organización, se calculará el VEGA, mediante la expresión 6:

$$
V E G A=\sqrt{I C_{\text {comp.SCI }} \times I C_{\text {proceso }}}
$$

Para la evaluación del indicador se tomarán los niveles de referencia mostrados en la tabla 1.

\section{Elaboración y discusión del informe de la evaluación}

Se elaborará el informe en función de las debilidades detectadas, donde el auditor deberá incluir como parte de su estudio sistemático, lo relativo a las sugerencias para incrementar la fiabilidad de los procesos críticos y en la evaluación de las normas del sistema de control interno. Los informes de los resultados deben incluir la descripción de los procedimientos empleados, la verificación de los resultados, así como el análisis del comportamiento histórico de los valores obtenidos y el análisis de su tendencia. 
Tabla 3

Pesos de cumplimiento de los indicadores

\begin{tabular}{lllll}
\hline Componentes & $\begin{array}{l}\text { Total de } \\
\text { indicadores }\end{array}$ & $\begin{array}{l}\text { Indicadores } \\
\text { correctos }\end{array}$ & $\begin{array}{l}\text { \% de indicadores } \\
\text { correctos }\end{array}$ & Normalizar \\
\hline Ambiente de control & 28 & 22 & 78.57 & 0.79 \\
Gestión y prevención de riesgos & 14 & 8 & 57.14 & 0.57 \\
Actividades de control & 16 & 9 & 56.25 & 0.56 \\
Información y comunicación & 7 & 3 & 52.86 & 0.43 \\
Supervisión y monitorización & 14 & 7 & 50.00 & 0.50
\end{tabular}

Fuente: elaboración propia.

\section{Fase IV. Mejora}

Objetivo: evaluar las actividades de supervisión y monitorización del control interno, bajo las pautas de la mejora continua.

\section{Comparación de los resultados}

Se compararán las tendencias (incremento, decremento, estacionalidad o estabilidad) del indicador con periodos anteriores. Se deberá profundizar en las causas que ocasionen variaciones en los valores de referencia del indicador.

\section{Elaboración del plan de acción}

Precisar el programa de implementación de los proyectos de mejora, para los cuales se establecerán los planes de acción o programas específicos, así como los medios técnicos y organizativos necesarios para su efectiva ejecución.

\section{Resultados}

El objetivo de este estudio fue determinar en la entidad hospitalaria seleccionada como objeto práctico, el VEGA, para esto se inició con la caracterización de la entidad que cuenta con elementos estratégicos pertinentes para este estudio, como una misión, visión y objetivos de trabajo, además de los resultados de la guía de autocontrol aplicada en julio del 2015, como resultado de una auditoría externa.

En la selección de los expertos para trabajar en la aplicación del procedimiento, se tuvo en cuenta, los criterios establecidos en este, quedando seleccionados los siguientes: director general, vicedirector de docencia, vicedirector de enfermería, vicedirector de administración, especialista del control interno, jefe dpto. centro oncológico y jefe dpto. atención al Grave. Se seleccionaron los procesos críticos y más importantes atendiendo a criterios como el impacto del proceso en la sociedad, costos generados, madurez del sistema de información y desempeño del proceso en la entidad mediante un voto ponderado, luego se realizó un análisis de Pareto (fig. 4) con sus pesos quedando como procesos pertinentes: esterilización, servicios de urgencias, consulta externa, hospitalización, egreso y atención a la población, donde existió un predominio de los procesos claves por su impacto en la sociedad.

Para este paso se revisó el resultado de la guía aplicada. La tabla 3 muestra los indicadores que fueron definidos satisfactoriamente por la organización, expresándose su porcentaje correspondiente, los que fueron normalizados. 


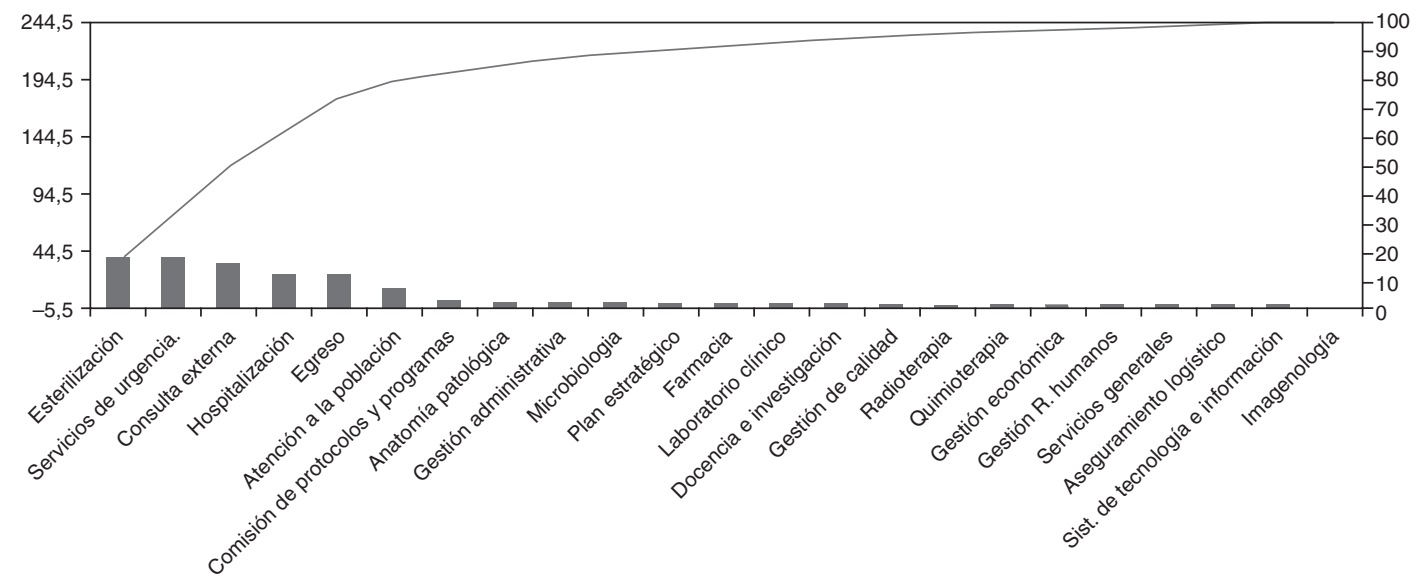

Figura 4. Representación de Pareto con los pesos de importancia de los procesos.

Fuente: elaboración propia. 
Tabla 4

Pesos de los componentes según expertos

\begin{tabular}{lccccccccc}
\hline Componentes & $\mathrm{E}_{1}$ & $\mathrm{E}_{2}$ & $\mathrm{E}_{3}$ & $\mathrm{E}_{4}$ & $\mathrm{E}_{5}$ & $\mathrm{E}_{6}$ & $\mathrm{E}_{7}$ & Total & Pesos \\
\hline Ambiente de control & 27 & 32 & 23 & 24 & 25 & 30 & 23 & 184 & 0.26 \\
Gestión y prevención de riesgos & 23 & 20 & 20 & 23 & 23 & 23 & 23 & 155 & 0.22 \\
Actividades de control & 10 & 16 & 20 & 10 & 10 & 15 & 11 & 92 & 0.13 \\
Información y comunicación & 15 & 22 & 21 & 25 & 17 & 22 & 25 & 147 & 0.21 \\
Supervisión y monitorización & 25 & 10 & 16 & 18 & 25 & 10 & 18 & 122 & 0.17
\end{tabular}

Estadísticos de contraste de la prueba de Fiedman

\begin{tabular}{ll}
\hline $\mathrm{N}$ & 5 \\
Chi-cuadrado & 1.157 \\
$\mathrm{gl}$ & 6 \\
Sig. asintót. & 0.979 \\
\hline
\end{tabular}

Fuente: elaboración propia.

Tabla 5

Determinación del índice de control por componente

\begin{tabular}{lllll}
\hline Proceso & $\mathrm{Ci}$ & $\mathrm{Pi}$ & $\mathrm{Ci} \times \mathrm{Pi}$ & \\
\hline Ambiente de control & 0.79 & 0.26 & 0.21 & \\
Gestión y prevención de riesgos & 0.57 & 0.22 & 0.13 & Evaluación \\
Actividades de control & 0.56 & 0.13 & 0.07 & 0.09 \\
Información y comunicación & 0.43 & 0.21 & 0.09 & Medio control \\
Supervisión y monitorización & 0.50 & 0.17 & & \\
Índice de gestión del control por componente & & &
\end{tabular}

Fuente: elaboración propia.

Se determinó el valor relativo a la importancia que se le atribuyó a la evaluación de un componente del control interno con respecto al resto. La tabla 4 visualiza el resultado de la encuesta realizada a los expertos que posibilitó determinar el peso de cada componente. Se verificó la concordancia de los expertos para lo cual se realizó la prueba de contraste de Friedman con la ayuda de los software SPSS versión 20.0 cuyo resultado muestra la tabla 4.

Según el resultado del cálculo en la tabla anterior: p-valor $=0.979>0.05$ por tanto, existió concordancia entre los expertos. Posteriormente se calculó el índice de gestión del control por componente como se aprecia en la tabla 5.

Para ello se encuestó a cada experto en una segunda vuelta, solicitándole que emitieran su opinión acerca de la importancia que tiene cada proceso seleccionado, con relación al resto de los procesos atendiendo a diferentes criterios como: evaluación mensual y anual, incremento de indisciplinas, madurez del sistema de información y desempeño del proceso. Los resultados se representan en la tabla 6 , de igual forma se verificó la concordancia de los expertos.

Según el resultado del cálculo en la tabla anterior: $p$-valor $=0.998>0.05$, existió concordancia entre los expertos. Seguidamente se procedió a representar la estructura y secuencias de los procesos. En la figura 5, se presenta la RdP que modela uno de los procesos seleccionado (servicio de urgencia) y en la tabla 7 se explica la leyenda de la RdP.

En la red, se evidencia la actividad en ejecución después de finalizado el proceso, en el caso de la tarea dos se disparará y se llegará al estado final estando en activación las tareas tres y cuatro respectivamente, así como fichas en sitios diferentes del sitio final, después de finalizado el proceso por el mismo suceso, por lo que el control en la tarea dos debe ser inminente. Lo antes 
Tabla 6

Pesos de los procesos según expertos

\begin{tabular}{lrrrrrrrrr}
\hline & $\mathrm{E}_{1}$ & $\mathrm{E}_{2}$ & $\mathrm{E}_{3}$ & $\mathrm{E}_{4}$ & $\mathrm{E}_{5}$ & $\mathrm{E}_{6}$ & $\mathrm{E}_{7}$ & Total & Pesos \\
\hline Esterilización & 35 & 30 & 35 & 30 & 20 & 32 & 35 & 217.00 & 0.31 \\
Servicios de urgencia & 20 & 25 & 20 & 25 & 20 & 18 & 18 & 146.00 & 0.21 \\
Consulta externa & 20 & 15 & 20 & 12 & 13 & 22 & 22 & 124.00 & 0.18 \\
Hospitalización & 12 & 10 & 12 & 13 & 12 & 15 & 12 & 86.00 & 0.12 \\
Egreso & 8 & 13 & 7 & 13 & 22 & 5 & 8 & 76.00 & 0.11 \\
Atención a la población & 5 & 7 & 6 & 7 & 13 & 8 & 5 & 51.00 & 0.07
\end{tabular}

Estadísticos de contraste de la prueba de Fiedman

\begin{tabular}{ll}
\hline $\mathrm{N}$ & 6 \\
$\mathrm{Chi}$-cuadrado & 0.524 \\
$\mathrm{gl}$ & 6 \\
Sig. asintót. & 0.998 \\
\hline
\end{tabular}

Fuente: elaboración propia.

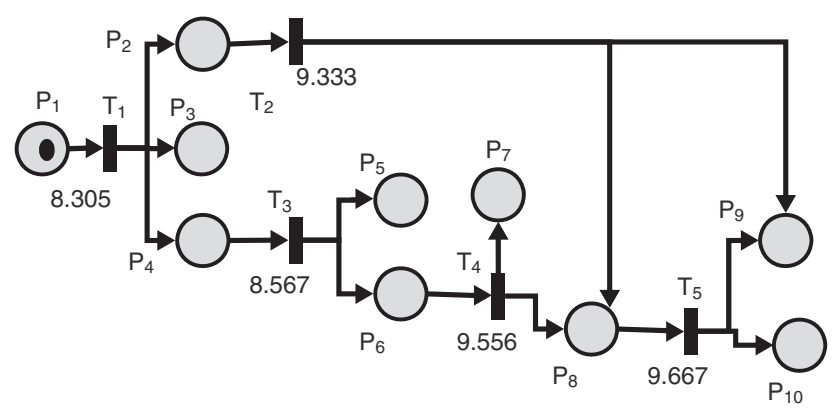

Figura 5. Red de Petri del proceso de servicio de urgencias.

Fuente: elaboracióm propia.

expuesto permite concluir que aunque el control está establecido en la mayoría de las actividades analizadas, se infiere la necesidad de incrementar este en la tarea anterior, pues en esta por la naturaleza de las decisiones, se incurrirían en altos gastos presupuestarios por concepto de tiempo, fuerza de trabajo y posibles pérdidas de vidas humanas.

Para el cálculo de la fiabilidad más probable, o sea el número de veces que no ocurrieron fallos en un horizonte temporal $\mathrm{dado}^{2}$, se tuvo en cuenta que en un mismo intervalo de tiempo determinado pueden ocurrir diferentes fallos, los datos se exponen en la tabla 8 .

$$
m_{T_{1}}=1-\frac{15}{90}=0.8333
$$

Por lo que la fiabilidad más probable de la tarea uno fue de un $83.33 \%$. El resto de las fiabilidades (optimista y pesimista) se determinaron mediante un cuestionario a los expertos en cada proceso.

\footnotetext{
${ }^{2}$ Se utilizó para este estudio el tercer trimestre del 2015.
} 
Tabla 7

Descripción de los elementos de la red de Petri

\begin{tabular}{|c|c|c|c|}
\hline \multicolumn{4}{|c|}{ Descripción de los elementos de la red } \\
\hline Lugares & Descripción & Transiciones & Descripción \\
\hline $\mathrm{P}_{1}$ & Paciente grave & $\mathrm{T}_{1}$ & $\begin{array}{l}\text { Clasificación del paciente y elaboración } \\
\text { del registro }\end{array}$ \\
\hline $\mathrm{P}_{2}$ & Paciente de atención inmediata & $\mathrm{T}_{2}$ & Cuidados intensivos y emergencias \\
\hline $\mathrm{P}_{3}$ & Libro de registro del paciente & $\mathrm{T}_{3}$ & $\begin{array}{l}\text { Comprobación de poder valerse por sí } \\
\text { solo }\end{array}$ \\
\hline $\mathrm{P}_{4}$ & Paciente sin atención inmediata & $\mathrm{T}_{4}$ & Consulta con el paciente \\
\hline $\mathrm{P}_{5}$ & Hoja de cargo de paciente en área de camilla & $\mathrm{T}_{5}$ & $\begin{array}{l}\text { Observación de la recuperación del } \\
\text { paciente }\end{array}$ \\
\hline $\mathrm{P}_{6}$ & Hoja de cargo de paciente en área de consulta & & \\
\hline $\mathrm{P}_{7}$ & Paciente de alta & & \\
\hline $\mathrm{P}_{8}$ & Paciente en observación & & \\
\hline $\mathrm{P}_{9}$ & Área especializada & & \\
\hline $\mathrm{P}_{10}$ & Paciente de alta & & \\
\hline
\end{tabular}

Fuente: elaboracióm propia.

Luego para calcular la fiabilidad de la tarea se procedió según lo planteado, obteniéndose que la fiabilidad de la tarea uno es de un $83.05 \%$, como se muestra a continuación:

$$
P_{r}=\frac{0.75+4 \times 0.8333+0,9}{6}=0.8305
$$

Tabla 8

Descripción de los fallos por actividades del proceso

\begin{tabular}{llll}
\hline Tareas & N. $^{\text {o de días con fallos }}$ & Descripción del fallo & N. $^{\text {o de repeticiones }}$ \\
\hline $\mathrm{T}_{1}$ & 15 & Falso pedido de materiales esterilizados & 6 \\
& & Utilización de historia clínica de pacientes no & 2 \\
& ingresados en hospital & 5 \\
& Demora en la atención al paciente & 2 \\
& No se han comprobado si se tienen los medios de & 3 \\
& & trabajo para prestar el servicio & \\
$\mathrm{T}_{2}$ & 6 & Apropiación indebida de medicamentos por & 2 \\
& & personal de farmacia, salas y servicios & 2 \\
& & Alteración resultados complementarios & \\
& & Roturas, extravíos y violaciones de normas técnicas & 2 \\
$\mathrm{~T}_{3}$ & & en el uso del equipamiento & 4 \\
& & Venta de servicios & 2 \\
& & Pedidos sin la debida jerarquización y autorización & 2 \\
& & Venta en el servicio de artículos u objetos & 4 \\
$\mathrm{~T}_{4}$ & & Maltrato & 2 \\
$\mathrm{~T}_{5}$ & & Favoritismo & 4 \\
& & Maltrato & 1 \\
& & Favoritismo & 2
\end{tabular}

Fuente: elaboración propia. 
Tabla 9

Diagnóstico de los cortes mínimos de la red

\begin{tabular}{lll}
\hline Cortes mínimos & Probabilidad de no fiabilidad & Cortes críticos \\
\hline $\mathrm{K}_{1}=\left(\mathrm{T}_{1}\right) ; \mathrm{K}_{2}=\left(\mathrm{T}_{2}\right) ; \mathrm{K}_{3}=\left(\mathrm{T}_{3}\right) ;$ & $\operatorname{Pr}\left(\mathrm{K}_{1}\right)=0.1695 ; \operatorname{Pr}\left(\mathrm{K}_{2}\right)=0.0667 ;$ & $\mathrm{K}_{1} ; \mathrm{K}_{2} ; \mathrm{K}_{3}$ \\
$\mathrm{~K}_{4}=\left(\mathrm{T}_{4} ; \mathrm{T}_{2}\right) ; \mathrm{K}_{5}=\left(\mathrm{T}_{5} ; \mathrm{T}_{2}\right)$ & $\operatorname{Pr}\left(\mathrm{K}_{3}\right)=0.1433 ;$ & \\
& $\operatorname{Pr}\left(\mathrm{K}_{4}\right)=0.0030 ;$ & \\
& $\operatorname{Pr}\left(\mathrm{K}_{5}\right)=0.0022 ;$ & \\
\end{tabular}

Fuente: elaboración propia.

Luego se determinaron los cortes críticos (tabla 9), como se supone independencia en los fallos, la probabilidad de que fallen ambos procedimientos es el producto de las no fiabilidades de las tareas que la componen. Para los cortes se seleccionaron aquellas actividades con probabilidad de no fiabilidad superiores al 5\% (este umbral fue fijado por el equipo auditor), en la tabla 9, se resume el diagnóstico de los cortes.

El cálculo de la fiabilidad del control del proceso (Ci) de servicio de urgencia quedó de la forma siguiente:

$$
C_{\text {Serviciode urgencia }}=1-\frac{3}{5}=0.4
$$

En el caso particular de este proceso la fiabilidad fue del $40 \%$, luego se identificó la fiabilidad (Ci) del resto de los procesos y se calculó el índice de control (IC) de la solución integral por proceso, como se muestra en la tabla 10.

Se determinó el VEGA del control interno, mediante sus dos componentes, quedando de la forma siguiente:

$$
V E G A_{S C I}=\sqrt{I C_{\text {componente }} \times I C_{\text {proceso }}}=\sqrt{0.58 \times 0.774}=0.67
$$

La gestión del control interno en la entidad fue de un 67\%, clasificando como alto, sin embargo no se considera eficiente atendiendo a que es una entidad hospitalaria, por lo que no se considera aceptable este VEGA. Para la elaboración del informe se tuvo en cuenta las debilidades detectadas en las tareas individuales en cada proceso, luego se discutió con los directivos de la entidad para la posterior elaboración del plan de acción, en función de sus debilidades, con su correspondiente responsable y ejecutores.

Tabla 10

\begin{tabular}{|c|c|c|c|c|}
\hline & $\mathrm{C}_{\mathrm{i}}$ & $P_{i}$ & $\mathrm{C}_{\mathrm{i}} \times \mathrm{P}_{\mathrm{i}}$ & \\
\hline Esterilización & 0.933 & 0.31 & 0.289 & \multirow{6}{*}{ Evaluación } \\
\hline Servicios de urgencia & 0.400 & 0.21 & 0.084 & \\
\hline Consulta externa & 0.867 & 0.18 & 0.156 & \\
\hline Hospitalización & 0.750 & 0.12 & 0.090 & \\
\hline Egreso & 0.800 & 0.11 & 0.088 & \\
\hline Atención a la población & 0.950 & 0.07 & 0.067 & \\
\hline \multicolumn{3}{|c|}{ Índice de gestión del control por proceso } & 0.774 & Alto control \\
\hline
\end{tabular}

Determinación del índice de control por proceso

Fuente: elaboración propia. 


\section{Discusión}

En las últimas décadas, la evaluación del control interno se realiza mediante la Guía de Autocontrol (Cuba) y diversos informes de control (otros países), que si bien son herramientas pertinentes, se limitan exclusivamente a la identificación de conformidades con los elementos asociados a sus componentes, la determinación de un valor de cumplimiento con las normas o índice de madurez del control interno como es el caso del modelo estándar del control interno en países latinoamericanos como Colombia con la Alcaldía Municipal de Gachancipa en el 2014. Por otra parte se revela la gestión por proceso mediante las ISO 9000:2015, donde se identifican carencias en su integración con el sistema de control interno, estudios recientes (Mar Cornelio et al., 2014), destacan la necesidad de la modelación multicriterio para el aumento de la efectividad en su evaluación, además de la propuesta de Andujar Rodríguez et al. (2000), de un algoritmo matemático para la detección de fallos del sistema de control interno donde no se evidencian las debilidades por componentes.

Es criterio de los autores, que la herramienta propuesta soluciona las carencias anteriores al permitir identificar en los procesos críticos, las actividades que los componen y componentes del control interno, los puntos críticos para este; con la utilización de la modelación multicriterio y las redes de Petri, como herramientas eficientes en la representación de procesos y detección de fallos. La utilización de estas contribuye a la determinación del VEGA y la elaboración de planes de acción objetivos y concretos, mediante las debilidades detectadas, a las cuales se enfocarán las soluciones integrales que impactan simultáneamente en varios elementos del control interno.

\section{Conclusiones}

Se comprobó la pertinencia de la modelación multicriterio y las redes de Petri, para la concepción y desarrollo del procedimiento propuesto, validándose en la práctica su factibilidad. Como resultado de la determinación del VEGA a partir del análisis por sus componentes y según los procesos de la entidad, se concluye que aunque la entidad hospitalaria objeto de estudio práctico presenta un control interno que se evalúa como alto, pues permite detectar las principales debilidades en su gestión, existen reservas que deben ser explotadas en aras de incrementar la eficiencia en sus servicios.

\section{Referencias}

Alverca Torres, G.X. y Valarezo Collahuazo, L.Y. (2012). Aplicación de las Redes de Petri en el dominio del cometimiento de hurto en supermercados.

Andujar Rodríguez, S. A., García Pérez, J. y Cruz Rembaud, S. (2000). Detección de las debilidades del Sistema de Control Interno en Auditoría: algoritmos matemáticos. Estudios de Economía Aplicada, 14, 5-36.

Araújo, R. T. S., Araújo, M. E. S., Medeiros, F. N. S. d. y Barroso, G. C. (2015). Modelagem de um sistema de gestão na Educação a Distância no Brasil utilizando redes de Petri Coloridas. Ingeniare. Revista chilena de ingeniería, 23(1), 145-158. http://dx.doi.org/10.4067/S0718-33052015000100016

Badri, S. A., Ghazanfari, M. y Shahanaghi, K. (2014). A multi-criteria decision-making approach to solve the product mix problem with interval parameters based on the theory of constraints. International Journal of Advanced Manufacturing Technology, 70(5-8), 1073-1080. http://dx.doi.org/10.1007/s00170-013-5360-8

Bolaño Rodríguez, Y. (2014). Modelo de dirección estratégica basado en la administración de riesgos para la integración del Sistema de Dirección de la Empresa. (Tesis presentada en opción al grado científico de Doctor en Ciencias Técnicas), Instituto Superior Politécnico José Antonio Echeverría, La Habana, Cuba.

Castro Rivera, S.A. y Cuervo Oliveros, J.S. A. (2015). Simulación de un proceso productivo en un ambiente de personalización masiva a través de redes de Petri. Trabajo de grado para optar por el título de Ingeniero Industrial, Universidad 
del Valle, Facultad de Ingeniería, Escuela de Ingeniería Industrial y Estadística, Ingeniería Industrial. Santiago de Cali.

Comas Rodríguez, R. (2013). Integración de herramientas de control de gestión para el alineamiento estratégico en el sistema empresarial cubano. Aplicación en empresas de Sancti Spíritus. (Tesis presentada en opción al grado científico de Doctor en Ciencias Técnicas). Matanzas: Universidad de Matanzas «Camilo Cienfuegos».

Distéfano, M. y Pérez, S. (2011). Desarrollo de sistemas concurrentes de control en tiempo real modelados con redes de petri. Ciencia y Tecnología, 3(1).

Hernández Cely, M. M., Leal, F. y López, J. (2013). Modelado e implementación de un sistemas automático de detección y diagnóstico de fallas basado en Redes de Petri para el proceso hogar en la generación de vapor. Scientia et Technica, 18(4), 599-606.

Mar Cornelio, O., Jiménez Hernández, R. C. y Bron Fonseca, B. (2014). Procedimiento para determinar el índice de control organizacional. Revista Infociencia, 18(2), 1-12.

Mejía Argueta, C., Gaytán Iniestra, J. y Arroyo López, M. d. P. E. (2014). Un enfoque multicriterio para el diseño de una red para el transporte de embarques internacionales. Contaduría y Administración, 59(4), $193-221$. http://dx.doi.org/10.1016/S0186-1042(14)70160-3

Morales Varela, A., Rojas Ramírez, J. A., Hernández Gómez, L. H., Morales González, Á. y Jiménez Reyes, M. Y. (2015). Modelo de un sistema de producción esbelto con redes de Petri para apoyar la toma de decisiones. Ingeniare. Revista chilena de ingeniería, 23(2), 182-195. http://dx.doi.org/10.4067/S0718-33052015000200004

Murillo Soto, L. D. (2008). Redes de Petri: Modelado e implementación de algoritmos para autómatas programables. Tecnología en Marcha, 21(4), 102-125.

Murillo Soto, L. D. (2010). Simulación de un sistema de manufactura flexible con redes de Petri coloreadas. Tecnología en Marcha, 23(1), 47-57.

Pérez Rave, J., Trujillo, M., Castro, G. y Gómez, G. (2014). Modelación multicriterio del nivel de prevención de contaminación por mercurio en entidades odontológicas. Ingeniare. Revista chilena de ingeniería, 23(1), $128-144$. http://dx.doi.org/10.4067/S0718-33052015000100015

Sánchez, S., Herrera, A. y Rovetto, R.C. A. (2014). Análisis y modelamiento del Canal de Panamá a través de las redes de petri. Artículo presentado en Proceedings of the 7th Euro American Conference on Telematics and Information Systems.

Zapata, G., Hoyos, B. y Quintero, L. (2014). Diseño del sistema automático para una planta piloto de recubrimientos electrolíticos. Parte I: Modelo mediante Redes de Petri Jerárquicas. Revista Facultad de Ingeniería, (45), 67-76. 